\title{
1 Investigating the Role of Chromatin Remodeler FOXA1 in Ferroptotic 2 Cell Death \\ 3
}

4 Emilie Logie ${ }^{1}$, Louis Maes ${ }^{1}$, Joris Van Meenen ${ }^{2}$, Peter De Rijk ${ }^{3,4}$, Mojca

5 Strazisar ${ }^{3,4}$, Geert Joris ${ }^{3,4}$, Bart Cuypers ${ }^{5}$, Kris Laukens ${ }^{5}$, Wim Vanden

6 Berghe $^{1^{*}}$

1. Laboratory of Protein Science, Proteomics and Epigenetic Signaling (PPES) and Integrated Personalized and Precision Oncology Network (IPPON), Department of Biomedical Sciences, University of Antwerp, Campus Drie Eiken, Universiteitsplein 1, Wilrijk, Belgium

2. Antwerp Research Group for Ocular Science (ARGOS), Department of Translational Neurosciences, University of Antwerp, Wilrijk, Belgium

3. Neuromics Support Facility, VIB Center for Molecular Neurology, VIB, Antwerp, Belgium

4. Neuromics Support Facility, Department of Biomedical Sciences, University of Antwerp, Antwerp, Belgium

5. Biomedical Informatics Network Antwerp (Biomina), Department of Computer Science, University of Antwerp, Wilrijk, Belgium

* Corresponding author: wim.vandenberghe@uantwerpen.be

Conflict of Interest: The authors declare no conflict of interest. 


\section{Investigating the Role of Chromatin Remodeler FOXA1 in Ferroptotic}

\section{Cell Death}

27 Ferroptosis is a lipid peroxidation-dependent mechanism of regulated cell death known to suppress tumor proliferation and progression. Although several genetic and protein hallmarks have been identified in ferroptotic cell death, it remains challenging to fully characterize ferroptosis signaling pathways and to find suitable biomarkers. Moreover, changes taking place in the epigenome of ferroptotic cells remain poorly studied. In this context, we aimed to investigate the role of chromatin remodeler forkhead box protein A1 (FOXA1) in RSL3-treated multiple myeloma cells because, similar to ferroptosis, this transcription factor has been associated with changes in the lipid metabolism, DNA damage, and epithelial-to-mesenchymal transition (EMT). RNA sequencing and Western blot analysis revealed that FOXA1 expression is consistently upregulated upon ferroptosis induction in different in vitro and in vivo disease models. In silico motif analysis and transcription factor enrichment analysis further suggested that ferroptosis-mediated FOXA1 expression is orchestrated by specificity protein 1 (Sp1), a transcription factor known to be influenced by lipid peroxidation. Remarkably, FOXA1 upregulation in ferroptotic myeloma cells did not alter hormone signaling or EMT, two key downstream signaling pathways of FOXA1. CUT\&RUN genome-wide transcriptional binding site profiling showed that GPX4-inhibition by RSL3 triggered loss of binding of FOXA1 to pericentromeric regions in multiple myeloma cells, suggesting that this transcription factor is possibly involved in genomic instability, DNA damage, or cellular senescence under ferroptotic conditions.

Keywords: FOXA1; forkhead box; ferroptosis; multiple myeloma; perichromatin

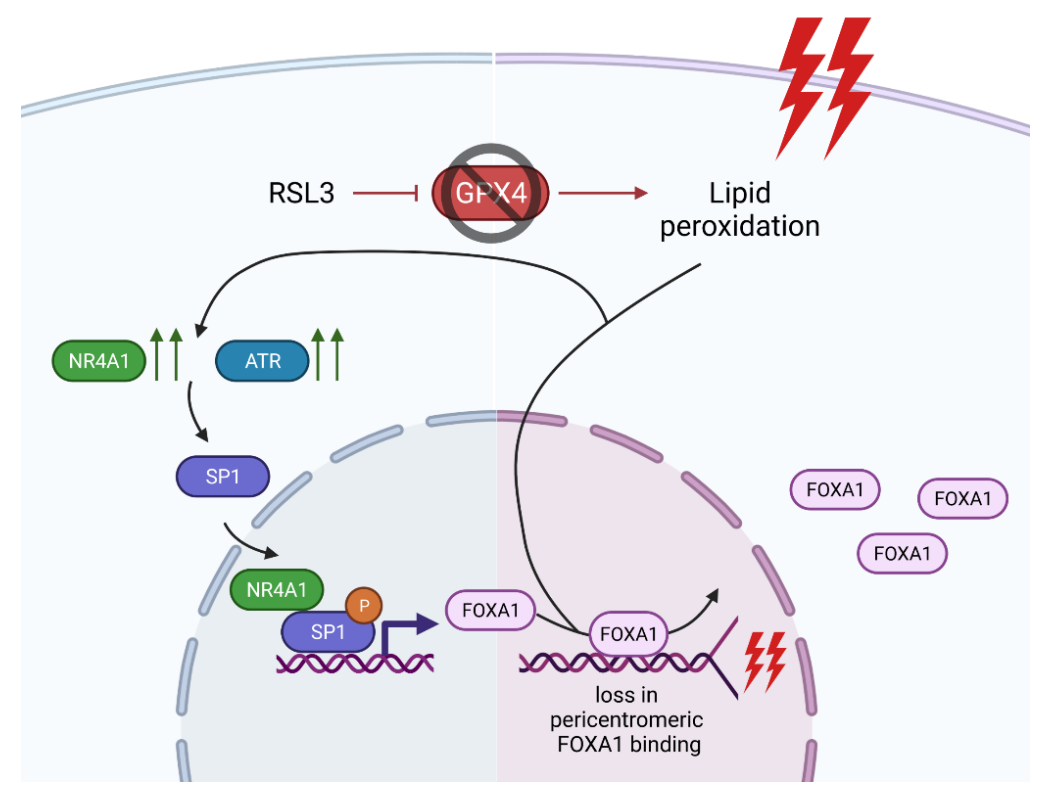




\section{Introduction}

50 Ferroptosis is a non-apoptotic mode of regulated cell death (RCD) characterized by an iron-dependent rise in reactive oxygen species (ROS) that propagate lipid peroxidation reactions [1]. Mechanistically, intracellular increases in labile ferrous iron $\left(\mathrm{Fe}^{2+}\right)$ challenge the cellular anti-oxidant defense systems by triggering the formation of toxic hydroxyl radicals through Fenton and Fenton-like chemistry [2]. Should enzymatic antioxidants, such as superoxide dismutase or glutathione peroxidases, fail to eliminate these toxic by-products, excessive peroxidation of polyunsaturated fatty acids (PUFAs) will ensue and ultimately cause detrimental loss of membrane integrity [3]. Several pathologies, including neurodegenerative diseases, cardiovascular diseases, ischemiareperfusion injuries, and diabetes, have already been associated with ferroptotic cell death [4-8]. Small molecules targeting ferroptosis signaling pathways have therefore gained considerable clinical interest in the past couple of years [9]. Interestingly, the induction of ferroptosis has also demonstrated to offer therapeutic potential, especially in the field of oncology. One of the major hallmarks of cancer cells includes evasion of apoptotic cell death due to (acquired) therapy resistance mechanisms [10]. Provoking non-apoptotic modes of cell death, such as ferroptosis or necroptosis, might therefore help in eliminating therapy-resistant cancer (stem) cells [11]. Additionally, compared to healthy tissue, malignant tumors heavily rely on an increased iron metabolism to sustain their augmented proliferation capacity, exposing them to higher basal levels of oxidative stress [12]. Further elevating intracellular $\mathrm{Fe}^{2+}$ concentrations with ferroptotic compounds might further disturb their precarious redox balance and efficiently promote cell death [13]. For example, several B-cell malignancies, including multiple myeloma (MM) and B-cell lymphomas, portray an increased iron uptake and display sensitivity to ferroptosis inducers [14-20].

On a molecular level, genetic and protein hallmarks of ferroptosis have been identified and are mainly involved in oxidative stress pathways (NRF2, GPX4, CHAC1) [17, 21, 22], iron metabolism (TFRC, FTH1) [23, 24], inflammation (PTGS2) [17], and lipid metabolism (ACSL4) [25]. The overexpression or downregulation of these genes have been considered as potential biomarkers of ferroptosis cell death, yet it remains challenging to find ferroptosis-specific markers [26]. ACSL4, for instance, is currently considered to be a specific driver for ferroptotic cell death as it is involved in enhancing 
82 [25, 27]. However, a recent study by Chu and colleagues has demonstrated that even

83 ACSL4-depleted cells can undergo p53-mediated ferroptosis [28]. Thus, there is an unmet need for finding more precise and specific contributors of ferroptotic cell death. A (combination of) suitable ferroptosis biomarker(s) might not only offer new insights in designing novel therapies for iron-related diseases, but might also aid in early detection of ferroptotic cells [29]. Moreover, it could help identify ferroptosis-resistant cancers, which, unfortunately, have already been identified as well [30-33]. In the present study, we investigated the role of chromatin remodeler forkhead box A1 (FOXA1) in MM cells undergoing ferroptotic cell death. FOXA1 belongs to a large family of FOX pioneer TFs that, unlike most TFs, can access target sequences located on nucleosomes and on some forms of compacted chromatin [34]. It is believed that members of the FOXA subfamily stably bind to genomic regions prior to activation and prior to binding of other TFs, and promote ATP-independent chromatin opening to allow binding of other TFs, nucleosome remodelers, or chromatin modifiers [34]. In case of FOXA1, it is suggested that chromatin opening is promoted by simultaneous DNA- and core histone binding (through a C-terminal domain), which disrupts local internucleosomal interactions required for stability of higher-order chromatin structure [35]. Depending on its chromatin recruitment sites, FOXA1 plays a role in embryonic development [35], hormone regulation [36, 37], lipid metabolism [38, 39], epithelial-tomesenchymal transition (EMT) [40, 41], and DNA damage [42]. Given that the three latter processes have directly been linked to ferroptosis sensitivity or ferroptotic cell death [27, 43, 44], we combined RNA and CUT\&RUN sequencing to characterize FOXA1 expression profiles and downstream targets in different ferroptosis models.

\section{Results}

\section{Ferroptotic Cell Death Promotes FOXA1 expression in Different Disease Models}

108 Although inhibition and induction of ferroptotic cell death is extensively being studied as

109 a therapeutic strategy in several disease models, finding suitable ferroptosis biomarkers

110 remains challenging [26]. To identify key genetic hallmarks of ferroptosis signaling

111 pathways, we compared publicly available RNAseq data (GSE104462) of erastin-treated

112 HEPG2 liver cancer cells to our own RNAseq data of RSL3-treated MM1 myeloma

113 cancer cells (awaiting GEO accession number). Despite considerable differences in

114 experimental design and starting material (Table 1), we found 23 common significant 
115 (FDR $<0.05 \&|\log 2 \mathrm{FC}>1|$ ) differentially expressed genes (DEGs) that displayed a

116 similar pattern in gene expression upon ferroptosis induction (Figure 1a). These genes are

117 mainly involved in metal binding (YPEL5, ZBTB10, MT2A, MT1F, MT1X), DNA

118 binding (BHLHE41, FOXA1, MAFF, KLF2, NR4A2), protein ubiquitination (HERPUD,

119 PELI1, FBXO32), calcium ion binding (STX11, JAG1), and protein dephosphorylation

120 (DUSP4, DUSP5). Interestingly, we could identify the ATP-independent chromatin

121 remodeler FOXA1 as one of the common genes between both RNAseq datasets. FOXA1

122 is a 473 amino acid long TF that belongs to the family of FOX pioneer TFs. Through its

123 winged forkhead domain (FKHD), it is able to open chromatin by disrupting

124 internucleosomal interactions (Figure 1b). In agreement with the RNAseq data, qPCR

125 and Western blot analysis revealed a time-dependent upregulation of FOXA1 expression

126 in therapy-resistant and - sensitive MM1 cells treated with RSL3, a class II ferroptosis

127 inducer (Figure 2a-c). As prolonged treatment with RSL3 results in decreased cell

128 viability, these data suggest that FOXA1 expression is tied to severity of ferroptotic cell

129 death and GPX4 inhibition (Figure 2b).

130 Given that we detected ferroptosis-mediated FOXA1 induction in two different cell types

131 (i.e. MM1 and HEPG2), we questioned whether similar observations could be made in in

132 vivo ferroptosis models. To this end, we performed Western blot analysis on liver samples

133 isolated from GPX4 liver-specific inducible knockout mice (Supplementary Figure S1).

134 LoxP-GPX4 homozygous mice carrying the cre transgene (Cre $\mathrm{Tg} /+$ ) demonstrated an

135 increased, yet not significant, FOXA1 protein expression compared to their healthy

136 controls $(\mathrm{Cre}+/+)$ (Figure $2 \mathrm{~d}$ ). Taken together, these findings suggest that FOXA1

137 upregulation may be a universal phenomenon in different ferroptotic (disease) models

138 and that FOXA1 might be a central regulator in ferroptosis signaling.

$140 \quad$ Table 1: Overview of experimental design differences in public RNAseq data vs own RNAseq data

\begin{tabular}{lcc}
\multicolumn{1}{c}{ Feature } & $\begin{array}{c}\text { Public RNAseq data } \\
\text { (GSE104462) }\end{array}$ & Our RNAseq data \\
\hline Cell line & HEPG2 & MM1S \& MM1R \\
\hline Tissue of origin & Liver & Peripheral blood \\
\hline Ferroptosis inducer & $\begin{array}{c}10 \mu \mathrm{M} \text { erastin } \\
\text { (inhibits Xc- system) }\end{array}$ & $\begin{array}{c}5 \mu \mathrm{M} \text { RSL3 } \\
\text { (inhibits GPX4) }\end{array}$ \\
\hline $\begin{array}{l}\text { Duration ferroptosis } \\
\text { treatment }\end{array}$ & $24 \mathrm{hr}$ & $3 \mathrm{hr}$ \\
\hline Ferroptosis inhibitor & $1 \mu \mathrm{M}$ ferrostatin-1 & $2 \mu \mathrm{M}$ ferrostatin-1 \\
\hline \multicolumn{2}{c}{ Abbreviations: Xc- system, Cystine/glutamate transporter; GPX4, Glutathione peroxidase 4. }
\end{tabular}




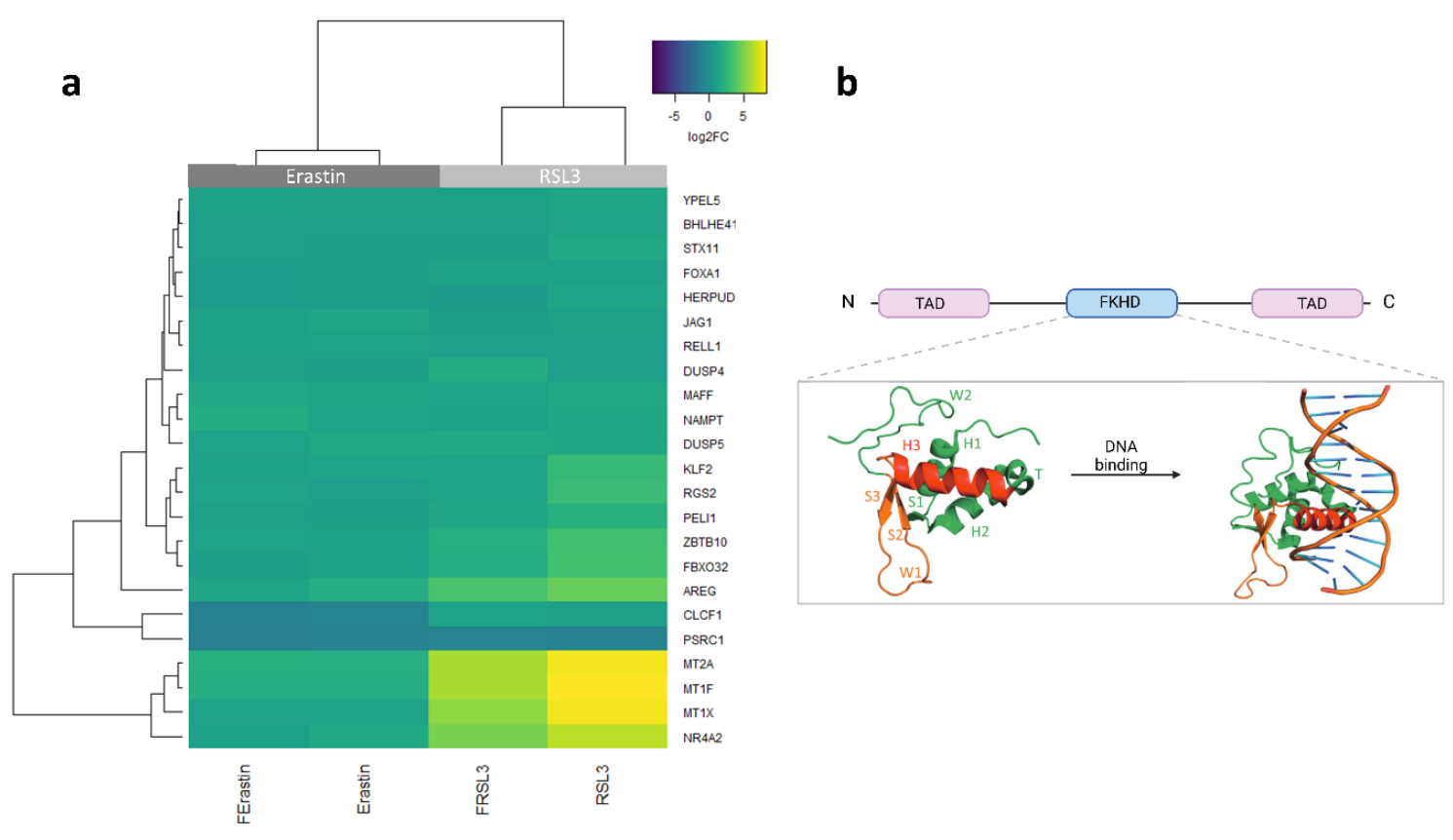

145 Figure 1: (a) Heatmap representation of common differentially expressed genes (FDR $<0.05, \log \mathrm{FC}>\mid 1$

146 |) between erastin-treated HEPG2 cells (publicly available data GSE104462) and RSL3-treated MM1 cells.

$147 N=3$ biologically independent replicates per cell line. (b) Schematic overview of Forkhead box A1

148 (FOXA1) protein domains. The forkhead domain (FKHD) is crucial for DNA binding and consists of $3 \alpha-$

149 helices (H1-3) and $3 \beta$-sheets (S1-3) organized in a helix-turn-helix motif. This motif is flanked on both

150 sides by polypeptide chain "wings" (W1-2) that interact with the minor DNA groove. 
a

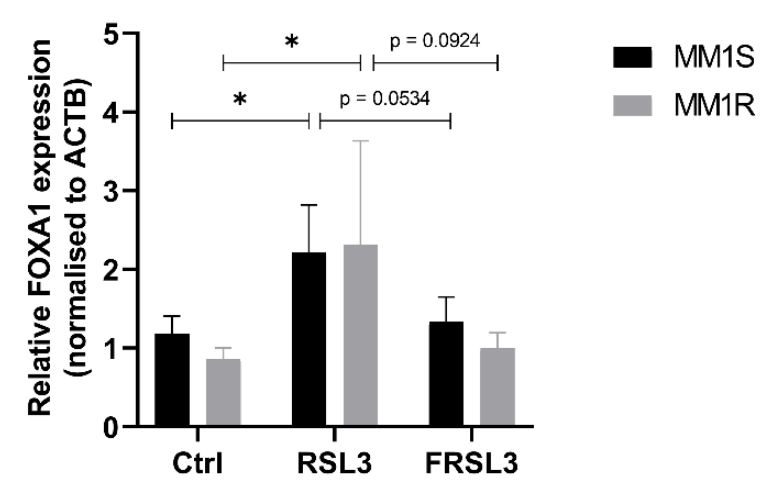

C

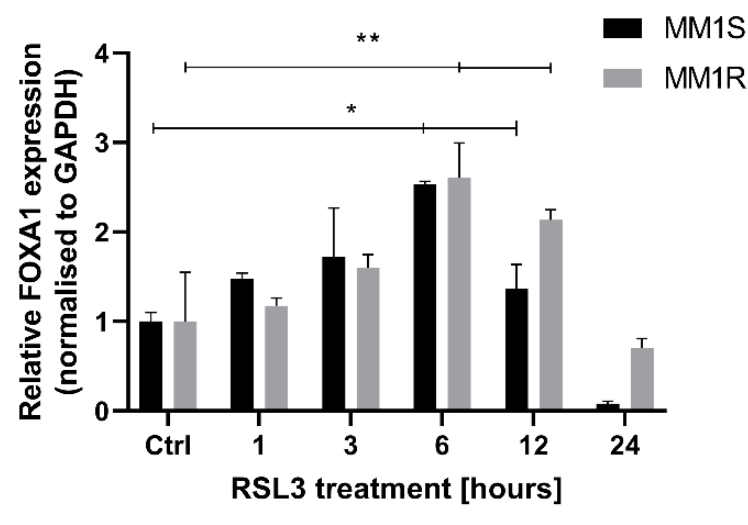

d

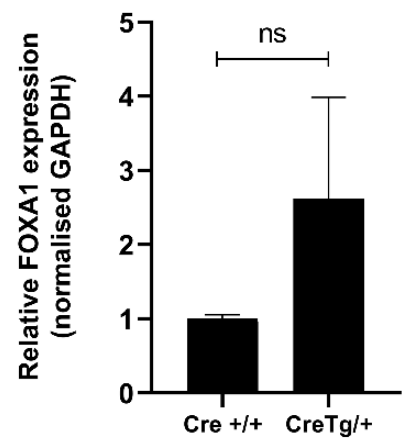

b

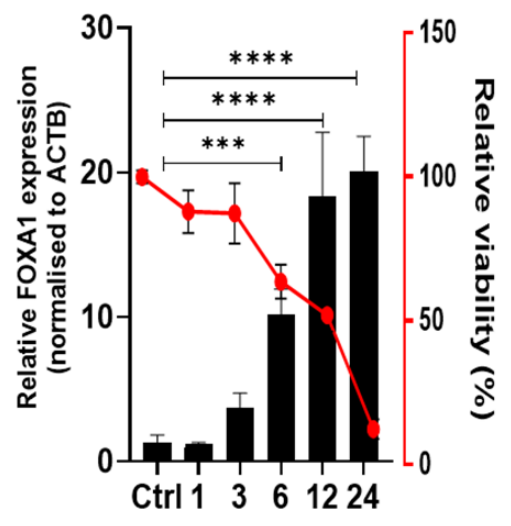

RSL3 treatment [hours]
Figure 2: (a) Relative FOXA1 mRNA expression in MM1R and MM1S cells treated with $5 \mu \mathrm{M}$ RSL3 for $3 \mathrm{hrs}$ with (FRSL3) or without (RSL3) $2 \mathrm{hr}$ pre-treatment with $2 \mu \mathrm{M}$ ferrostatin-1 compared to untreated controls. FOXA1 expression is normalized against the $\beta$-actin (ACTB) housekeeping gene. Data are plotted as the mean \pm s.d., $n=3$ biologically independent samples per cell line (*p < 0.05), ANOVA). (b) Relative mRNA FOXA1 expression and cell viability (\%) in MM1 cells after RSL3 treatment. FOXA1 expression is normalized against ACTB mRNA expression. Data are plotted as the mean \pm s.d., $n=3$ biologically independent samples per cell line $(* * * \mathrm{p}<0.001, * * * * \mathrm{p}<0.0001$, ANOVA). (c) Western blot detection and quantification of FOXA1 and GAPDH expression levels in MM1 cells treated with RSL3. Data are plotted as the mean \pm s.d., $n=3$ biologically independent samples. (d) Western blot detection and quantification of FOXA1 and GAPDH expression levels in liver samples from healthy Cre $+/+$ mice versus sick Cre $\mathrm{Tg} /+$ mice. Data are plotted as the mean \pm s.d., $n=2 \mathrm{Cre}+/+$ mice and $3 \mathrm{Cre} \mathrm{Tg} /+$ mice $(\mathrm{ns}=\mathrm{p}>$ 0.05 , two-tailed t-test). 

Conditions

167 A PubMed search of all articles featuring the FOXA1 transcription factor revealed that FOXA1 expression is mostly associated with hormone signaling in prostate, breast and testis cancer (Supplementary Figure S2). Therefore, RNAseq data was further explored to assess whether expression of nuclear hormone receptors is significantly altered in ferroptotic MM1 cells. Supplementary Figure S3 demonstrates that most hormone receptors, including estrogen (ESR), glucocorticoid (NR3C1), retinoid X (RXR), and peroxisome proliferator-activated receptors (PPAR) remain largely unaltered upon RSL3 treatment. Similarly, we could not detect significant differences in ferroptosis sensitivity in glucocorticoid-sensitive MM1S cells, expressing NR3C1, versus glucocorticoidresistant MM1R cells, lacking functional NR3C1 expression. In contrast, an increase in mRNA expression of lipid and oxidative metabolism sensing orphan nuclear receptors NR4A1, NR4A2, and NR4A3 could be observed in RSL3-treated cells compared to untreated controls, and was partly validated by Western blot (Supplementary Figure S4).

180 The interplay between FOXA1 and orphan nuclear receptors has only poorly been characterized, mostly in context of dopaminergic neurons [45, 46]. Interestingly, important tumor suppressor roles for NR4A TFs have recently been described (reviewed in [47]), and NR4A defects are reported to promote formation of blood-tumors (e.g. leukemia, lymphoma) and T-cell immunity dysfunctions [48-51]. As such, possible antitumor functions of NR4A TFs in ferroptotic cells deserves further investigation.

We next explored whether FOXA1 might play a role in epithelial-to-mesenchymal transition (EMT) as reported in previous studies [40, 41, 52]. Correlation analysis of the RNAseq data indeed demonstrated that genes highly correlated with FOXA1 expression were enriched in cytoskeleton organization, epithelial cell differentiation, and regulation of EMT (Figure 3a-c). Interestingly, the EMT status is known to directly affect ferroptosis sensitivity, with mesenchymal cells being more susceptible to ferroptotic cell death compared to epithelial cells [43]. Ferroptosis-mediated upregulation of FOXA1 might subsequently drive MM1 cells towards a mesenchymal profile and promote cell death by RSL3. To this end, qPCR analysis of four key EMT markers was performed on MM1 cells treated with RSL3 for increasing timepoints (Supplementary Figure S5). Overall, no significant expression differences of epithelial marker E-cadherin (E-CAD) or mesenchymal markers N-cadherin (N-CAD), Twist-related protein 1 (TWIST1) or Snail 
199 preliminary results indicate that FOXA1 does not orchestrate trans-differentiation of

200 MM1 cells into a mesenchymal phenotype.

201 Since our targeted approaches did not further elucidate the role of FOXA1 in ferroptosis

202 signaling, we aimed to characterize the downstream effects of FOXA1 by performing

203 CUT\&RUN sequencing. This technique allows for genome-wide profiling of chromatin

204 binding sites of transcription factors, similar to ChIP-Seq [53]. In short, MM1R cells were

205 treated for 3 hours with $5 \mu \mathrm{M}$ RSL3, after which FOXA1-bound DNA fragments were

206 collected and purified for downstream analysis. After completing library preparation and

207 DNA sequencing, enriched regions were called using the sparse enrichment analysis for

208 CUT\&RUN (SEARC) [54]. Only a limited number of genomic regions $(n=43)$ were

209 identified to be differentially altered in FOXA1 binding after RSL3 treatment

210 (Supplementary Table S2). Although we previously measured higher FOXA1 expression

211 in ferroptotic cells, untreated controls displayed higher FOXA1 binding compared to

212 RSL3-treated cells (Supplementary Table S2). Remarkably, all identified regions were

213 located in pericentromeric DNA (Figure 4a-b), suggesting that a ferroptosis-mediated loss

214 of FOXA1 binding to pericentromeric hetero-chromatin takes place upon RSL3

215 induction. Given that FOXA1 is a pioneer TF that is able to bind compact DNA, these

216 observations could indicate that DNA decondensation (of pericentromeric regions)

217 triggers genome-wide loss of FOXA1 binding. In line with these results, we previously

218 found (unpublished data) that ferroptosis might induce early cellular senescence in MM1

219 cells, a process which has been associated with defective pericentric silencing and

220 decondensation [55, 56]. Preliminary Western blot analysis of RSL3-treated MM1R cells

221 confirmed the observed loss of FOXA1 expression in chromatin-bound cellular protein

222 fractions (Figure 5). In parallel, cytoplasmic protein expression of FOXA1 was slightly

223 increased upon RSL3 exposure, indicating that chromatin-free FOXA1 is transported

224 toward the cytoplasmic compartment (Figure 5). 
a

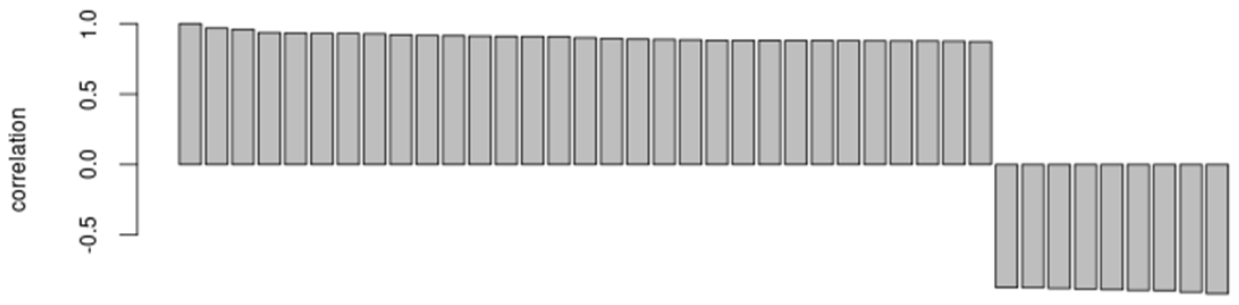

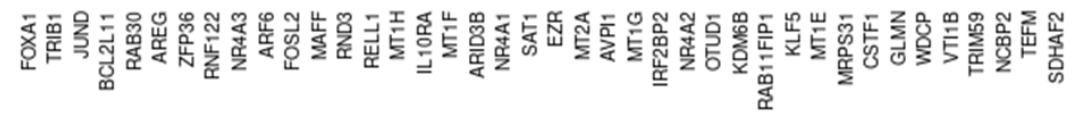

b

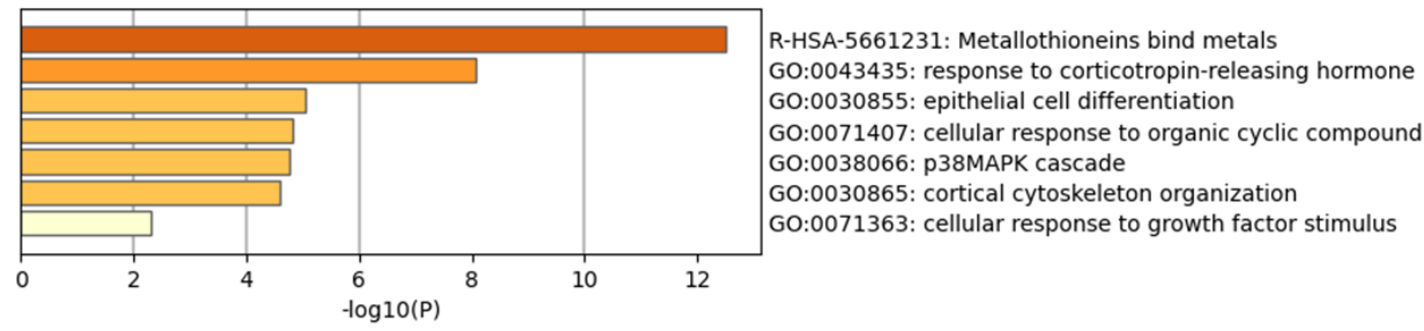

C

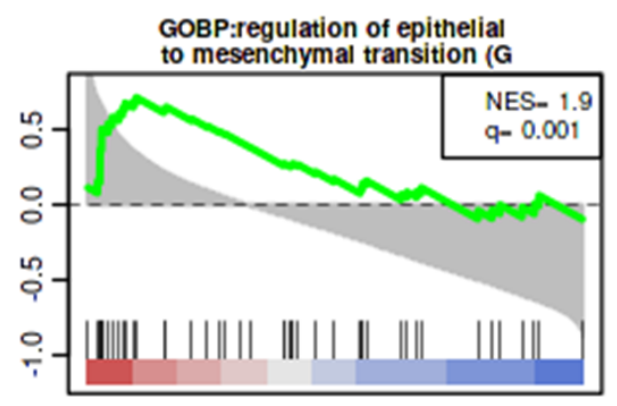

\begin{tabular}{lll}
\hline Gene & Rho & \multicolumn{1}{c}{ Title } \\
\hline FOXA1 & 1,00 & forkhead box A1 \\
\hline LDLRAD4 & 0,71 & $\begin{array}{l}\text { low density lipoprotein receptor class A domain-containing } \\
\text { protein 4 }\end{array}$ \\
\hline TGFB2 & 0,67 & transforming growth factor beta 2 \\
\hline SDCBP & 0,67 & syndecan binding protein \\
\hline COL1A1 & 0,67 & collagen type I alpha 1 chain \\
\hline SMAD7 & 0,65 & SMAD family member 7 \\
\hline TGFBR1 & 0,65 & transforming growth factor beta receptor 1 \\
\hline BMP2 & 0,56 & bone morphogenetic protein 2 \\
\hline BAMBI & 0,52 & BMP and activin membrane bound inhibitor \\
\hline CTNNB1 & 0,45 & catenin beta 1 \\
\hline PPP2CA & 0,42 & protein phosphatase 2 catalytic subunit alpha \\
\hline SMAD3 & 0,41 & SMAD family member 3 \\
\hline TGFB3 & 0,36 & transforming growth factor beta 3 \\
\hline STRAP & 0,34 & serine/threonine kinase receptor associated protein \\
\hline
\end{tabular}

STRAP 0,34 serine/threonine kinase receptor associated protein

Figure 3: (a) Histogram plot displaying the top correlated genes in respect to FOXA1. The height of the bars corresponds to the Pearson correlation value. Figure was generated using the Omics Playground tool (v2.7.18). (b) Metascape pathway analysis [57] of RNAseq data displaying the top 20 significantly enriched pathways of RSL3-treated MM1 cells compared to untreated controls. (c) Functional GSEA enrichment of genes correlated with FOXA1 expression (left). The green curve corresponds to the normalized enrichment score (NES). Black vertical bars indicate the rank of genes in the gene set in the sorted correlation metric. FDR is represented by the q-value in the figure. Figure was generated using the Omics Playground tool (v2.7.18). The leading-edge table (right) reports the leading edge genes as reported by GSEA corresponding to the selected geneset. The 'Rho' columns report the correlation with respect to FOXA1.

\section{Sp1 is a Possible Driver of FOXA1 Expression}

238 Taking into account that FOXA1 is upregulated under different ferroptotic conditions in

239 different cell lines, we investigated whether a common transcription factor drives

240 expression of FOXA1. To this end, we generated a list of potential FOXA1 driver genes

241 by identifying transcription factor binding sites located in the FOXA1 promotor region 
242 obtained from the SwissRegulon database [58]. Next, a target list of each of the candidate

243 drivers was constructed using three different databases, namely IFTP, TRRUSR, and

244 Marbach2016, employed in the tftarget R package [59]. Finally, an overlap between

245 candidate driver target genes and significant DEGs identified in the two RNAseq studies

246 was performed. Our analysis showed that transcription factor Sp1 is the most probable

247 driver in FOXA1 expression, both in MM1R cells and HEPG2 cells (Table 2).

Table 2: Top 5 candidate drivers of FOXA1 expression in ferroptotic cells.

\begin{tabular}{ccc}
\hline $\begin{array}{c}\text { Candidate } \\
\text { driver }\end{array}$ & $\begin{array}{c}\text { \# DEGs in RNAseq data } \\
\text { regulated by candidate } \\
\text { driver }\end{array}$ & $\begin{array}{c}\text { \% DEGs in RNAseq } \\
\text { regulated by candidate } \\
\text { driver }\end{array}$ \\
\hline Sp1 & 46 & 82.14 \\
\hline SPI1 & 36 & 64.29 \\
\hline TFAP2A & 33 & 58.93 \\
\hline TFAP2C & 31 & 55.36 \\
\hline RREB1 & 30 & 55.57 \\
\hline
\end{tabular}
alpha; TFAP2C, Transcription factor AP-2 gamma; RREB1, Ras responsive element binding protein 1

253 Although other studies have reported a ferroptosis-dependent increase in Sp1 expression

254 [7, 60, 61], we did not find significant alterations in Sp1 mRNA levels in RSL3-treated

255 MM1 cells compared to untreated controls (Figure 6a). Nonetheless, preliminary

256 experiments demonstrate that siRNA silencing of $\mathrm{Sp} 1$ abolishes ferroptosis-driven

257 FOXA1 upregulation (Figure 2a, Figure 6b), suggesting that Sp1 (partly) drives FOXA1

258 upregulation in MM1 cells.

259 Possibly, ferroptotic triggers regulate Sp1 transcriptional activity through alternative

260 mechanisms and subsequently promote downstream FOXA1 expression. In agreement

261 with this hypothesis, a recent kinome screen has revealed that $\mathrm{Sp}-1$ upstream ATR

262 damage response serine/threonine kinase directly impacts ferroptosis sensitivity [62]. Sp1

263 phosphorylation is known to directly impact Sp-1 dependent transcription [63] and might

264 be increased in ferroptotic cells. Alternatively, ferroptosis signaling pathways could 265 increase expression of Sp1 cofactors and promote Sp1 target site binding. NR4A1 is a 266 cofactor of Sp1 that has recently been described in ferroptotic cell death [64] and was 267 also found to be upregulated in this study. Through its interaction with Sp1, NR4A1 might 268 recruit Sp1 more efficiently to its GC-ich gene targets and promote transcription [65].

269 Further research exploring the Sp1-FOXA1 signaling axis during ferroptosis are needed

270 to fully confirm the role of Sp1 in RSL3-dependent FOXA1 expression. 
bioRxiv preprint doi: https://doi.org/10.1101/2021.10.13.461056; this version posted October 14,2021 . The copyright holder for this preprint (which was not certified by peer review) is the author/funder, who has granted bioRxiv a license to display the preprint in perpetuity. It is made available under aCC-BY-ND 4.0 International license.

a

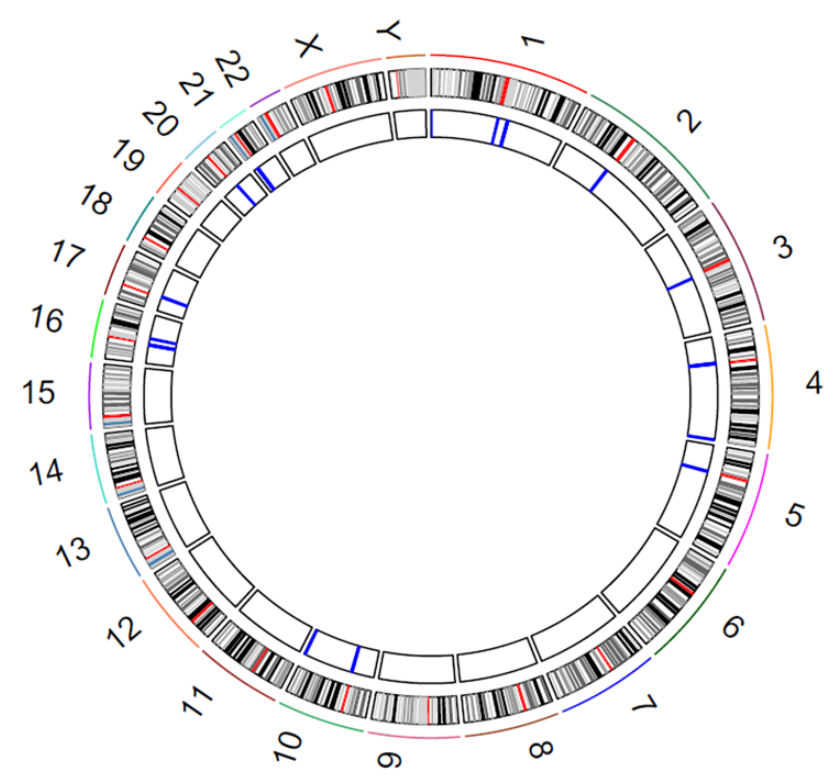

b

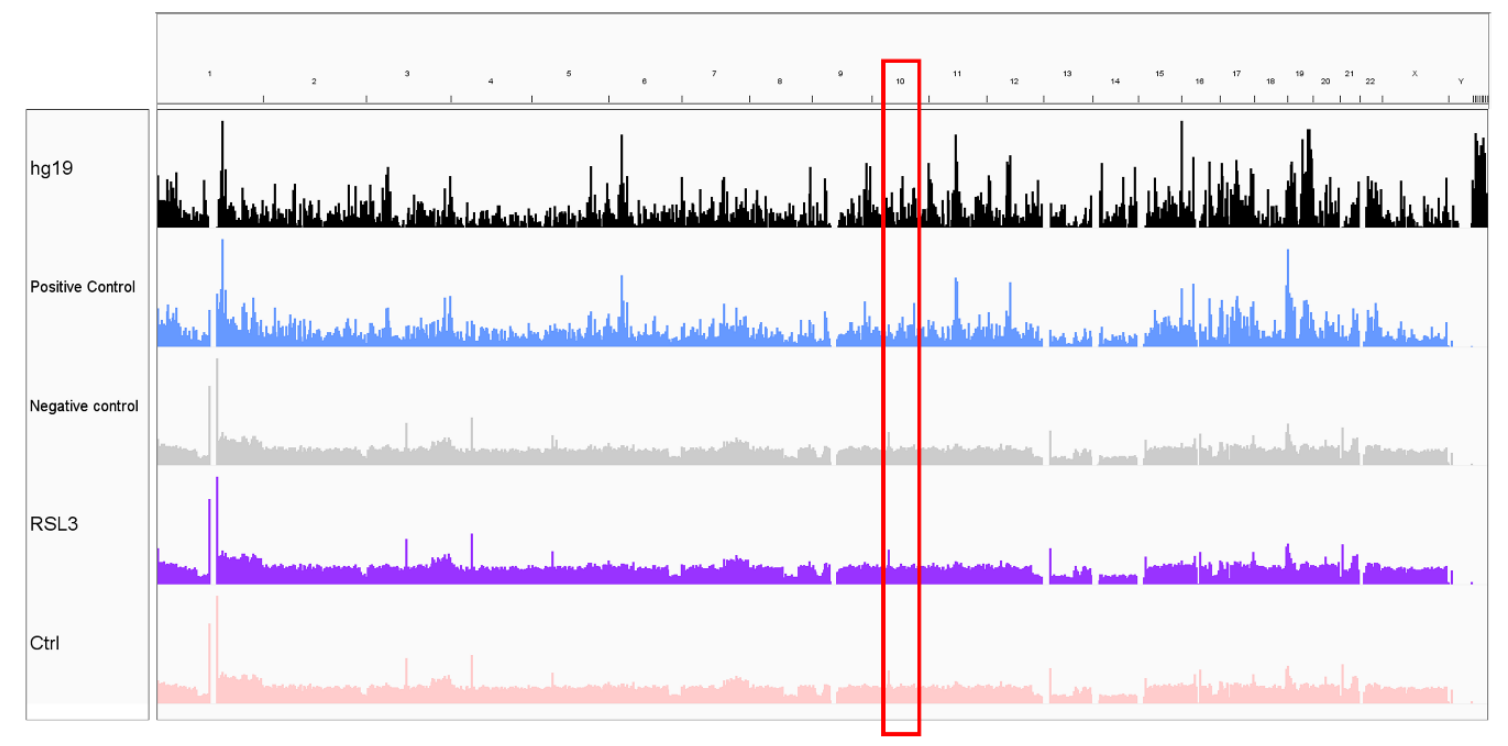

chr10: 41,861 - 41,790 kb

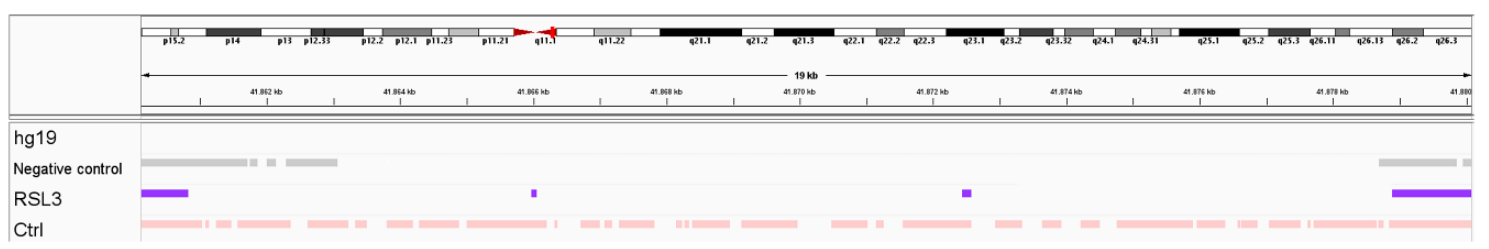

Figure 4: (a) Circos plot displaying chromosome ideograms (outer ring) with centromeric regions marked in red. Blue markings on the inner ring show the differentially enriched FOXA1-bound DNA regions in untreated control cells compared to RSL3-treated cells. (b) Overview of genomic regions sequenced in each CUT\&RUN treatment condition (upper panel). The hg19 panel represents the reference genome and displays known mapped genes. The lower panel represents a close-up visualization of chr10: 41,861 $41,790 \mathrm{~kb}$ highlighting the loss of FOXA1 binding to perichromatin in RSL3-treated cells compared to controls. Figures were generated with Integrative Genomics Viewer (v2.9.4). 


\section{Discussion}

281 In the past decade, RCD and ferroptosis research has grown rapidly, especially in the field of neurological diseases and oncology [66]. Several morphological, biochemical, genetic, and protein hallmarks of ferroptotic cell death have been identified over the last years, but the exact executioner signals of ferroptosis remain largely unknown (reviewed in [29]). Identification of specific ferroptosis contributors may therefore provide novel opportunities for creating anti-cancer therapies. Consequently, we compared RNAseq data of ferroptotic MM and HEPG2 cells to explore whether common expression signatures could be found in these different experimental setups. Our analysis revealed that genes involved in metal binding, DNA binding, protein ubiquitination, and protein phosphorylation were shared in both ferroptosis models. Of particular interest, we identified ATP-independent chromatin remodeler FOXA1 to be specifically upregulated upon RSL3 and erastin treatment. FOXA1 levels were also found to be upregulated in liver tissue obtained from liver-specific GPX4 inducible knock-out mice, suggesting that increased FOXA1 mRNA and protein expression might be a universal trigger in various ferroptosis disease models. Further in silico motif and TF enrichment analysis predicted that Sp1 is the most likely driver of ferroptosis-driven FOXA1 expression. Sp1 has previously been described in context of lipid peroxidation and ferroptotic cell death, and is hypothesized to play a dual role in the regulation of tissue injury [7, 60, 61]. However, our qPCR data demonstrate that Sp1 expression remains unaltered in RSL3-treated MM1 cells, implying that transcriptional activity of Sp1 is orchestrated through other upstream mechanisms. Post-translational modifications (PTMs), such as protein phosphorylation, are reported to directly influence Sp1 activity and might be altered in ferroptotic conditions [67]. Indeed, several upstream kinases responsible for Sp1 phosphorylation, including p38 and ATM/ATR are known to be involved in ferroptosis signaling as well [62, 68-70]. Alternatively, transcription activity of Sp1 may be stimulated through improved recruitment to its DNA target binding sites by cofactor proteins that are differentially expressed in the presence of ferroptotic stimuli. NR4A1, for example, has recently been identified as a modulator of ferroptotic cell death and is also reported to act

309 as a cofactor of $\mathrm{Sp} 1$ [64, 65]. Follow-up proteomics, Western blot, and

310 immunoprecipitation experiments will undoubtedly reveal to which extend PTMs and 311 cofactor-recruitment of Sp1 are crucial for FOXA1 expression.

312 Two independent studies have recently reported that nuclear hormone receptor activity is

313 highly correlated with ferroptosis sensitivity [71, 72]. Presumably, cells with higher 

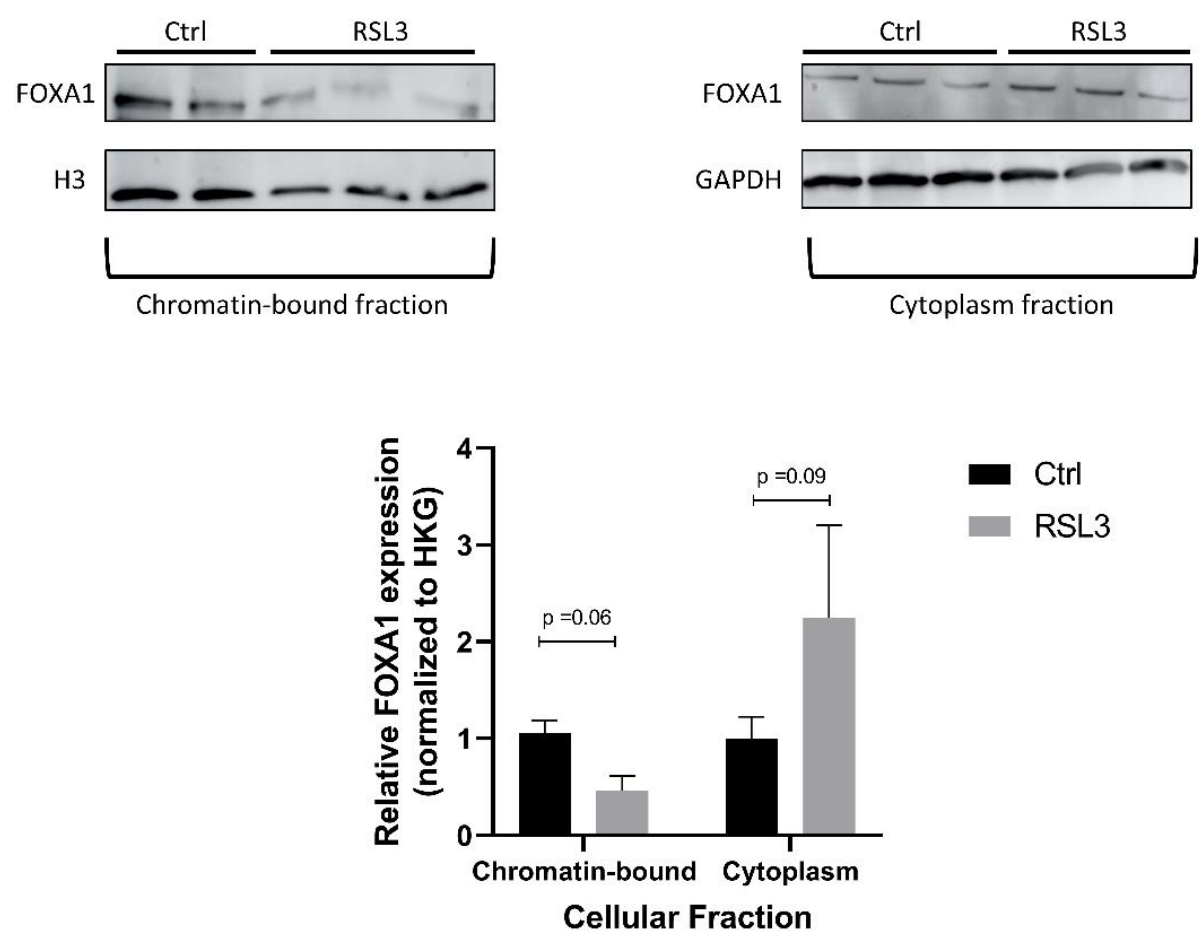

Figure 5: Relative FOXA1 protein expression of different cellular protein fractions in MM1R cells treated with $5 \mu \mathrm{M}$ RSL3 compared to untreated controls. Data are plotted as the mean \pm s.d., $n=3$ independent samples per treatment (indicated p-values are outcomes of unpaired, two-tailed t-test).

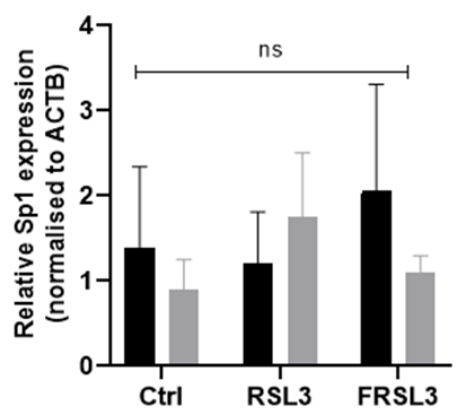

b
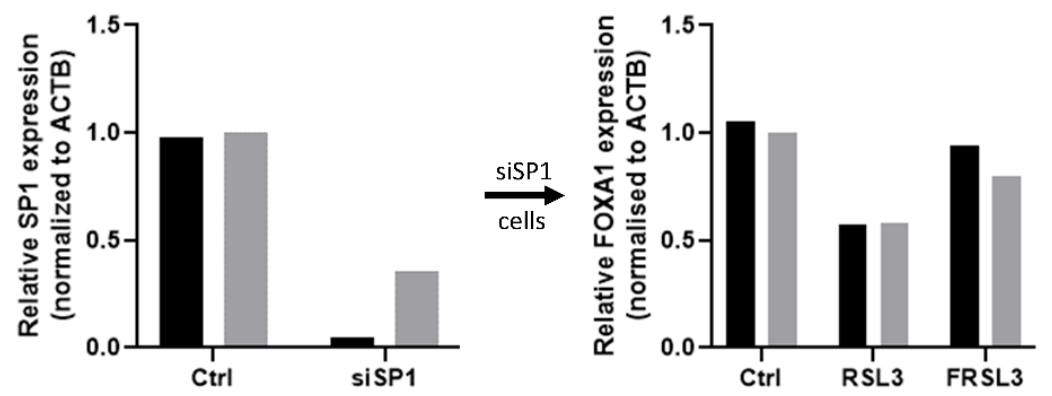

Figure 6: (a) Relative mRNA Sp1 expression in MM1 cells after $3 \mathrm{hr}$ treatment with $5 \mu \mathrm{M}$ RSL3, with (FRSL3) or without pre-treatment with ferrostatin. Data are plotted as the mean \pm s.d., $n=3$ biologically independent samples per cell line (ns $=\mathrm{p}>0.05$, ANOVA). (b) Relative mRNA SP1 (left) and FOXA1 (right) expression in MM1 cells transfected with siRNA targeting SP1. Data are plotted as the mean \pm s.d., 
endocrine activity are subjected to hormone-dependent ROS production, which promotes lipid peroxidation through activation of Fenton reactions [73]. Because FOXA1 is a critical interacting partner of several nuclear receptors [74], we wondered whether ferroptosis induction in MM1 cells is associated with an increase in hormone receptor activity. A preliminary screening of hormone receptor expression mRNA changes in RSL3-treated MM1 cells showed that the expression of the majority of nuclear receptors remain unchanged. Only a subset of orphan nuclear receptors, NR4A1-3, are specifically upregulated upon RSL3 induction. Although FOXA1 has been reported to regulate NR4A2 expression in immature midbrain dopaminergic neurons [45], the interplay between both proteins needs to be explored further. Possibly, NR4A receptors mediate ferroptotic cell death by influencing the cellular energy and lipid metabolism [64, 75]. On the other hand, these orphan receptors might orchestrate ferroptosis signaling pathways by recruiting other ferroptosis-dependent proteins to their target site, as previously explained. NR4A orphan receptors have also been associated with tumor suppressor functions, and mutations in NR4A1-3 have been linked with the formation of blood cancers, including leukemia and lymphoma [48, 49]. RSL3-driven upregulation of NR4A

341 TFs might therefore drive elimination of MM cancer cells by regulating key cancer pathways (reviewed in [47]. Intriguingly, both ferroptosis and NR4A proteins are known to be regulated by the p53 tumor suppressor $[76,77]$ and indicates that an GPX4-NR4Ap53 signaling network may drive MM cell death. Further research about the anti-cancer effects of NR4A TFs in ferroptotic cells could potentially offer new therapeutical insights for $\mathrm{MM}$ and other hematological malignancies. Regardless, based on assessing expression changes, FOXA1 does not seem to primarily target (steroid) hormone receptors during ferroptosis. A direct measurement of hormone receptor activity, by evaluating nuclear translocation or by performing ChIP for example, might aid in fully characterizing the effects of FOXA1 in hormone signaling. Furthermore, evaluating FOXA1-dependent changes on nuclear receptors in more endocrine active cell systems, such as breast or pancreas cancer cell lines, might reveal cell type-dependent effects of FOXA1.

354 Because both FOXA1 and ferroptosis have been associated with EMT [40, 43, 52], we also investigated whether RSL3 treatment triggers significant changes in EMT markers.

356 Generally, (tumor) cells harboring a more mesenchymal profile are considered to portray an increased ferroptosis sensitivity because they heavily rely on GPX4 activity compared to their epithelial counterparts [78]. Mesenchymal-state cells also exhibit more 
dysregulated antioxidant programs, explaining why ferroptotic compounds are more potent in these cells $[78,79]$. In this regard, ferroptosis-dependent reprogramming of the epithelial-mesenchymal state through FOXA1 upregulation, might promote ferroptosis sensitivity. While our RNAseq data revealed a correlation of FOXA1 expression with several other drivers of EMT, including TRIB1, N-CAD, E-CAD, SLUG, and TWIST1 mRNA expression was not significantly altered upon RSL3 incubation. This suggests that MM1 cells do not shift toward a more epithelial - or mesenchymal-like state under ferroptotic conditions.

367 Given that neither hormone signaling or EMT seem to be direct downstream targets of FOXA1 in ferroptotic MM1 cells, genome-wide transcription site profiling was performed by CUT\&RUN. Similar to ChIP-Seq, this technique combines ChIP with parallel DNA sequencing to identify binding sites of DNA-associated proteins, such as FOXA1. Unfortunately, signal-to-noise signals were quite low in our treatment setups, with signal intensities being similar to the negative $\operatorname{IgG}$ control. Further optimization of the experimental setup is therefore required before biologically relevant interpretations can be finalized. Increasing the starting amount of MM1 cells or addition of an extra cross-linking step might improve experimental outcome, especially since FOXA1 has been reported to transiently bind to its DNA sites [80]. Taking this into account, we could still identify 43 genome regions wherein FOXA1 binding was significantly altered in RSL3-treated MM1 cells compared to their untreated controls. Remarkably, all these regions were located in pericentromeric chromatin and FOXA1 binding was significantly lower in RSL3 conditions, despite the earlier observed transcriptional and translational FOXA1 upregulation in MM1 cells. Pericentric (satellite) DNA is typically considered to be void of functional genes and transcriptionally silent since they are confined in transcriptionally inert heterochromatin [81]. However, mounting evidence suggests that pericentric transcripts are crucial in maintaining genome stability (reviewed in [82] and [83]). To this end, loss of FOXA1 binding in ferroptotic cells could potentially promote genome instability and DNA double strand breakage. Another possibility is that ferroptotic stress triggers defective pericentric transcription due to dramatic DNA decondensation, as is also observed when cells are exposed to UV (i.e. DNA damage), cadmium toxicity or cellular senescence [56, 84-86]. Given that FOXA1, as a pioneer TF, mainly binds to heterochromatin regions, genome-wide DNA decondensation might promote overall loss of FOXA1 pericentromeric DNA binding and uncontrolled pericentric transcription. Our previous work (unpublished data) has indeed suggested that 
393 ferroptosis is associated with an epigenomic stress response linked to oxidative stress and cellular senescence, suggesting that DNA decondensation might occur in ferroptotic cells. Intriguingly, FOXA1 expression has been reported to increase with cellular senescence

396 [87]. Possibly, loss binding to heterochromatin pericentromeric DNA promotes 397 recruitment of FOXA1 to other target sites that trigger cellular senescence [87]. Repeating 398 the CUT\&RUN experiments under optimized experimental conditions should help in 399 investigating this hypothesis further. Alternatively, "DNA-free" FOXA1 might localize 400 to the cytoplasm and inhibit nuclear translocation of other TFs to promote cell death [88].

401 This seems to occur in our experimental setup as well, given that Western blot analysis 402 revealed an RSL3-dependent increase in FOXA1 expression in cytoplasmic protein 403 fractions.

404 Taken together, our data suggest that ferroptosis triggers a time-dependent upregulation of FOXA1 expression in different experimental models, which could be orchestrated by transcriptional activation of Sp1. The downstream effects of this FOXA1 expression surge in MM1 cells remain somewhat elusive but do not seem to include steroid hormone signaling or EMT. In contrast, preliminary data imply that ferroptotic stress might trigger uncontrolled pericentric transcription and genome instability, due to loss of FOXA1binding to pericentromeric DNA. Moreover, relocalization of pericentric-free FOXA1 to secondary target sites or the cytoplasm might further promote cellular stress responses, such as cellular senescence or cell death.

\section{Materials and Methods}

\section{Cell Culture and Cell Viability Assays}

416 Human MM1S cells (CRL-2974) and MM1R cells (CRL-2975) were purchased from 417 ATCC. RPMI-1640 medium, supplemented with 10\% FBS (E.U Approved; South 418 American Origin) and 1\% Pen-Strep solution (Invitrogen, Carlsbad, CA, USA), was used 419 to sustain the cells. The cells were cultivated at $37^{\circ} \mathrm{C}$ in $5 \% \mathrm{CO} 2$ and $95 \%$ air atmosphere 420 and $95-98 \%$ humidity. To assess cell viability, the colorimetric assay with 3-(4, 5421 dimethylthiozol-2-yl)-2, 5-diphenyltetrazolium bromide (MTT) was used (Sigma 422 Aldrich, St. Louis, MO, US) as previously described [89].

\section{Antibodies and Reagents}

424 RSL3 was purchased from Selleckchem (Houston, USA), dissolved in DMSO and stored 425 as $50 \mathrm{mM}$ stocks at $-20^{\circ} \mathrm{C}$. siRNA targeting $\mathrm{Sp} 1$ (1299001) was purchased from 

Antibodies FOXA1 (ab23738) and GAPDH (2118S) were obtained from Abcam (Cambridge, UK) and Cell Signaling Technology (Danvers, MA, USA), respectively.

\section{RNA Extraction and Sequencing}

430 After cell harvest, total RNA from untreated or RSL3-treated (with or without $2 \mathrm{hr}$ pre431 treatment with $2 \mu \mathrm{M}$ Ferrostatin-1) MM1S and MM1R cells was extracted using the 432 RNeasy Mini Kit (Qiagen, Venlo, the Netherlands) according to the manufacturer's 433 protocol. Once isolated, quantification was performed with the Qubit RNA BR Assay Kit 434 (ThermoFisher, MA, USA) and RNA was stored at $-80^{\circ} \mathrm{C}$. Extracted RNA was used as input for RNA sequencing as previously described [90]. In brief, RNA was shipped to 436 BGI (BGI Group, Bejing, China) where quality checks were performed using the 4372100 Bioanalyzer system (Agilent Technologies, USA) and sequencing took place using 438 the BGISE-500 platform (BGI Group, Beijing, China). Quality control, genome mapping 439 and differential gene expression analysis was performed using the R440 packages FastQC (v0.11.5) [91], STAR (v2.7.3a) [92], and DESeq2 (v3.12) [93]. DEGs 441 were considered to be significant when $\mathrm{FDR}<0.05$ and $|\log 2 \mathrm{FC}|>1$. Raw gene counts 442 from the GSE104462 dataset [94] were extracted from the Gene Expression Omnibus 443 (GEO) database and used as input for the same RNAseq analysis pipeline as described 444 above.

\section{cDNA Synthesis and Quantitative Real-time PCR}

446 Extracted RNA from RSL3-treated cells was converted into cDNA using the Go-

447 Script reverse transcription system (Promega, Madison, Wisconsin, USA) according to 448 the manufacturer's protocol. Subsequently, qPCR analysis was carried out using 449 the GoTag qPCR Master Mix (Promega, Madison, Wisconsin, USA) as explained 450 by manufacturer's protocol. In short, $1 \mu \mathrm{L}$ cDNA was added to a master mix comprising 451 SYBR green, nuclease-free water, and $0.4 \mu \mathrm{M}$ forward and reverse primers. The 452 following PCR program was applied on the Rotor-Gene Q qPCR machine (Qiagen, 453 Venlo, the Netherlands): $95^{\circ} \mathrm{C}$ for 2 min, 40 cycli denaturation $\left(95^{\circ} \mathrm{C}, 15 \mathrm{~s}\right)$ and 454 annealing/extension $\left(60^{\circ} \mathrm{C}, 30 \mathrm{~s}\right)$, and dissociation $\left(60-95^{\circ} \mathrm{C}\right)$. Each sample was run in 455 triplicate and the median value was used to determine the $\Delta \Delta \mathrm{Ct}$-values using $\beta$-actin 456 (BACT) as the normalization gene. Primer sequences are listed in Supplementary Table 457 S1. 


\section{Protein Extraction and Western blot Analysis}

459 Cellular protein extraction occurred by resuspending cell pellets in $0.5 \mathrm{~mL}$ RIPA buffer 460 (150 mM NaCl, 0.1\% Triton X-100, 1\% SDS, $50 \mathrm{mM}$ Tris-HCl pH 8) supplemented 461 with PhosphataseArrest (G-Biosciences, Saint-Louis, MO, USA) and protease inhibitors 462 (Complete Mini®, Roche). After $15 \mathrm{~min}$ incubation on ice with regular vortexing, 463 samples were briefly sonicated (1 min, amplitude $30 \mathrm{kHz}$, pulse $1 \mathrm{~s}$ ) and centrifuged at $46413200 \mathrm{rpm}$ for $20 \mathrm{~min}$ at $4^{\circ} \mathrm{C}$. Solubilized proteins were transferred to new Eppendorf 465 tubes and stored at $-20{ }^{\circ} \mathrm{C}$. To extract proteins from mouse liver tissue, RIPA buffer 466 containing 2\% SDS was added to the tissue. Sample homogenisation was performed 467 with the TissueRuptor, followed by 1 hour incubation at $4{ }^{\circ} \mathrm{C}$ on a rotor. Sonication ( 5 $468 \mathrm{~min}$, low amplitude $1 \mathrm{kHz}$ and $20 \mathrm{~Hz}$ burst rate) was used to shear DNA and debris was 469 removed by centrifugation for $8 \mathrm{~min}$ at $13000 \mathrm{~g}$.

470 Using standard protocols, all protein samples were separated using Bis-Tris SDS471 PAGE with a high-MW MOPS running buffer, and transferred onto nitrocellulose 472 membranes (Hybond C, Amersham) using the Power Blotter System (Thermofisher, MA, 473 USA). Blocking the membranes for 1 hour with blocking buffer ( $20 \mathrm{mM}$ Tris-HCl, 140 $474 \mathrm{mM} \mathrm{NaCl}, 5 \% \mathrm{BSA}, \mathrm{pH} 7,5)$ at RT was followed by overnight incubation with the 475 primary antibody at $4^{\circ} \mathrm{C}$. Blots were then incubated for $1 \mathrm{hr}$ with the secondary, HRP 476 dye-conjugated antibody (Dako, Glostrup, Denmark) after which 477 chemiluminescent signals were detected with the Amersham Imager 680 (Cytiva, MA, 478 USA) and quantified with the ImageJ software (v1.53j) [95].

\section{Liver Samples of Cre-lox Liver-Specific GPX4 Knockout Mice}

480 Liver samples from Cre-lox liver-specific inducible GPX4 kockout mice were kindly 481 provided by Ines Goetschalckx and Prof. Dr. Tom Vanden Berghe (Laboratory of 482 Pathophysiology, University of Antwerp). GPX4 knockout mice were generated by 483 crossing homozygous GPX4-floxed mice with heterozygous GPX4 conditional knockout 484 mice (Supplementary Figure S1).

\section{Nucleofection of MM cells}

486 MM1 cells were transfected using the Nucleofector IIb device (Lonza Amaxa, 487 Switzerland) as described by the manufacturer's protocol. Briefly, 1 million cells were 488 resuspended in $100 \mu \mathrm{L}$ supplemented nucleofector solution. Next, $300 \mathrm{nM}$ siSp1 was 489 added to resuspended cells. To assess transfection efficiency, an additional pmaxGFP 490 Vector (Lonza, Bazel, Switserland) was included in each nucleofection reaction (average 
transfection efficiency $=51.3 \pm 2.4 \%$ ). Cell suspensions were transferred to a provided cuvettes and nucleofection was performed using the O-020 program. 48 hours after transfection, cells were harvested and used as input for qPCR analysis.

\section{Motif Analysis and Transcription Factor Enrichment Analysis}

495 To search for a potential driver of FOXA1, a list of candidate drivers was composed using 496 transcription factor binding sites from the SwissRegulon database located at 497 the FOXA1 promoter [58, 96]. Using that list, a list of targets of these candidate drivers 498 was composed using data from three different databases - IFTP, TRRUST and 499 Marbach2016 - provided via the tftargets package in R [59]. Targets of each candidate 500 driver were matched to DEGs common to both datasets and two metrics for overlap were 501 calculated: the number of overlapping genes and the percentage of overlap with respect to the number of DEGs. As an additional control, the X2Kweb tool was further used to identify putative enriched transcription factors through Transcription factor enrichment analysis (TFEA) [97]. Results from TFEA were compared with results from IFTP, TRRUST and Marbach2016.

\section{CUTANA Cut\&Run to Identify Chromatin-Associated Proteins}

Downstream targets of FOXA1 were identified using the EpiCypher CUTANA ChIC CUT\&RUN Kit (23614-1048, EpiCypher, USA) as previously described [53, 98]. In short, $5 \times 10^{5}$ MM1R were plated into 6-well plates and either treated with $5 \mu \mathrm{M}$ RSL3 (3 hours) or left untreated. For each treatment condition, 4 biological replicates were included. Cells were washed and bound to concanavalin A-coated magnetic beads and permeabilized with wash buffer (20 mM HEPES pH 7.5, $150 \mathrm{mM} \mathrm{NaCl}, 0,5 \mathrm{mM}$ spermidine and protease inhibitors) supplemented with 0,05\% digitonin. After overnight incubation at $4^{\circ} \mathrm{C}$ with the primary FOXA1 antibody (13-2001, Epicypher), cell-bead slurry was washed twice more after which pA-MNase digestion was activated by placing samples on an ice-cold block and incubated with digitonin wash buffer containing $2 \mathrm{mM}$ $\mathrm{CaCl}$. Each CUT\&RUN experiment also featured a positive (anti-H3K4me3) and negative (anti-Rabbit IgG) antibody control. After 2 hours, the cleavage reaction was stopped with stop buffer (340 mM NaCl, 20 mM EDTA, 4 mM EGTA, 0.05\% digitonin, $0.05 \mathrm{mg} / \mathrm{mL}$ glycogen, $5 \mu \mathrm{g} / \mathrm{mL}$ RNase A, $2 \mathrm{pg} / \mathrm{mL}$ E. coli spike-in DNA) and fragments were released by 30 -minute incubation at $37^{\circ} \mathrm{C}$. Samples were centrifuged and DNAcontaining supernatant was collected. DNA extraction was performed with a DNA 
524 library preparation using Kapa HypePrep Kit (7962363001, Roche) and barcoding using

525 xGen UDI-UMI barcodes (10005903, IDT) following manufacturers protocols.

526 Barcoded libraries of ten samples (4 treated, 4 untreated and two controls) were

527 equimolarly pooled and sequenced on MiSeq (Illumina) using MiSeq v3 150 reagent kit

528 (MS-102-3001, Illumina) according to manufacturer's protocol. The run ended in

529 obtaining $4.16 \mathrm{~Gb}, 56.66$ million reads (Q30 92.09\%, 3,86 Gb).

530 Sequencing data were aligned to the UCSC h38 reference genome using the Burrows-

531 Wheeler Aligner [99] and peaks were called using SEARC [54] after conversion to the

532 bedgraph format using bedtools [100]. Peaks were merged using Granges [101]. For each

533 peak region, the number of mapping reads was counted using chromVAR [102]

534 getCounts. E.coli spike-in counts were obtained by alignment to the eschColi_K12 E.coli

535 reference. Differential analysis was performed using the DESeq2 package (v3.12) [93],

536 where counts were normalized to E.coli spike-in counts. Differentially enriched regions

537 were visualized with the RCircos R package (v1.2.1) [103] and IGV (v2.9.4)

538 (BroadInstitute, Cambridge, MA, USA).

\section{Subcellular Protein Fractionation}

540 The subcellular protein fractionation kit (\# 78840, Thermofisher, MA, USA) was used to

541 fractionate proteins into nuclear and cytoplasmic fractions according to the

542 manufacturer's instructions. The yield of obtained chromatin-bound nuclear proteins and

543 cytoplasmic proteins was determined by the BCA method. Finally, $20 \mu \mathrm{g}$ of protein from

544 each cellular fraction was used to perform SDS-PAGE and Western blot analysis, as

545 described above.

$546 \quad$ Statistical Analysis

547 Statistical tests were performed in GraphPad Prism (v7.0) (GraphPad Software, San

548 Diego, CA, USA) unless otherwise stated in the main text. Results were considered to be 549 statistically significant when p-values $<0.05$ were obtained. 


\section{$551 \quad$ References}

552 1. Dixon SJ, Lemberg KM, Lamprecht MR, Skouta R, Zaitsev EM, Gleason CE, Patel DN, Bauer AJ, Cantley AM, Yang WS, et al. Ferroptosis: an iron-dependent form of nonapoptotic cell death. Cell. 2012;149(5):1060-72.

2. Stockwell BR, Friedmann Angeli JP, Bayir H, Bush AI, Conrad M, Dixon SJ, Fulda S, Gascon S, Hatzios SK, Kagan VE, et al. Ferroptosis: A Regulated Cell Death Nexus Linking Metabolism, Redox Biology, and Disease. Cell. 2017;171(2):273-85.

3. Yan B, Ai Y, Sun Q, Ma Y, Cao Y, Wang J, Zhang Z, Wang X. Membrane Damage during Ferroptosis Is Caused by Oxidation of Phospholipids Catalyzed by the Oxidoreductases POR and CYB5R1. Mol Cell. 2021;81(2):355-69 e10.

4. Kupershmidt L, Amit T, Bar-Am O, Weinreb O, Youdim MB. Multi-target, neuroprotective and neurorestorative M30 improves cognitive impairment and reduces Alzheimer's-like neuropathology and age-related alterations in mice. Mol Neurobiol. 2012;46(1):217-20.

5. Yan N, Zhang J. Iron Metabolism, Ferroptosis, and the Links With Alzheimer's Disease. Front Neurosci. 2019;13:1443.

6. Fang X, Wang H, Han D, Xie E, Yang X, Wei J, Gu S, Gao F, Zhu N, Yin X, et al. Ferroptosis as a target for protection against cardiomyopathy. Proc Natl Acad Sci U S A. 2019;116(7):267280.

7. Li Y, Feng D, Wang Z, Zhao Y, Sun R, Tian D, Liu D, Zhang F, Ning S, Yao J, et al. Ischemiainduced ACSL4 activation contributes to ferroptosis-mediated tissue injury in intestinal ischemia/reperfusion. Cell Death Differ. 2019;26(11):2284-99.

8. Li S, Zheng L, Zhang J, Liu X, Wu Z. Inhibition of ferroptosis by up-regulating Nrf2 delayed the progression of diabetic nephropathy. Free Radic Biol Med. 2021;162:435-49.

9. Han C, Liu Y, Dai R, Ismail N, Su W, Li B. Ferroptosis and Its Potential Role in Human Diseases. Front Pharmacol. 2020;11:239.

10. Fernald K, Kurokawa M. Evading apoptosis in cancer. Trends Cell Biol. 2013;23(12):620-33.

11. Hassannia B, Vandenabeele P, Vanden Berghe T. Targeting Ferroptosis to Iron Out Cancer. Cancer Cell. 2019;35(6):830-49.

12. Torti SV, Torti FM. Iron and Cancer: 2020 Vision. Cancer Res. 2020;80(24):5435-48.

13. Trachootham D, Alexandre J, Huang P. Targeting cancer cells by ROS-mediated mechanisms: a radical therapeutic approach? Nat Rev Drug Discov. 2009;8(7):579-91.

14. VanderWall K, Daniels-Wells TR, Penichet M, Lichtenstein A. Iron in multiple myeloma. Crit Rev Oncog. 2013;18(5):449-61.

15. Steegmann-Olmedillas JL. The role of iron in tumour cell proliferation. Clin Transl Oncol. 2011;13(2):71-6. for future treatments. J Exp Clin Cancer Res. 2019;38(1):406. 
17. Yang WS, SriRamaratnam R, Welsch ME, Shimada K, Skouta R, Viswanathan VS, Cheah JH, Clemons PA, Shamji AF, Clish CB, et al. Regulation of ferroptotic cancer cell death by GPX4. Cell. 2014;156(1-2):317-31.

18. Bordini J, Morisi F, Cerruti F, Cascio P, Camaschella C, Ghia P, Campanella A. Iron Causes Lipid Oxidation and Inhibits Proteasome Function in Multiple Myeloma Cells: A Proof of Concept for Novel Combination Therapies. Cancers (Basel). 2020;12(4).

19. Zhong Y, Tian F, Ma H, Wang H, Yang W, Liu Z, Liao A. FTY720 induces ferroptosis and autophagy via PP2A/AMPK pathway in multiple myeloma cells. Life Sci. 2020;260:118077.

20. Kinowaki Y, Kurata M, Ishibashi S, Ikeda M, Tatsuzawa A, Yamamoto M, Miura O, Kitagawa M, Yamamoto K. Glutathione peroxidase 4 overexpression inhibits ROS-induced cell death in diffuse large B-cell lymphoma. Lab Invest. 2018;98(5):609-19.

21. Abdalkader M, Lampinen R, Kanninen KM, Malm TM, Liddell JR. Targeting Nrf2 to Suppress Ferroptosis and Mitochondrial Dysfunction in Neurodegeneration. Front Neurosci. 2018;12:466.

22. Dixon SJ, Patel DN, Welsch M, Skouta R, Lee ED, Hayano M, Thomas AG, Gleason CE, Tatonetti NP, Slusher BS, et al. Pharmacological inhibition of cystine-glutamate exchange induces endoplasmic reticulum stress and ferroptosis. Elife. 2014;3:e2523.

23. Feng H, Schorpp K, Jin J, Yozwiak CE, Hoffstrom BG, Decker AM, Rajbhandari P, Stokes ME, Bender HG, Csuka JM, et al. Transferrin Receptor Is a Specific Ferroptosis Marker. Cell Reports. 2020;30(10):3411-23.e7.

24. Park E, Chung SW. ROS-mediated autophagy increases intracellular iron levels and ferroptosis by ferritin and transferrin receptor regulation. Cell Death Dis. 2019;10(11):822.

25. Yuan H, Li X, Zhang X, Kang R, Tang D. Identification of ACSL4 as a biomarker and contributor of ferroptosis. Biochem Biophys Res Commun. 2016;478(3):1338-43.

26. Tang D, Chen X, Kang R, Kroemer G. Ferroptosis: molecular mechanisms and health implications. Cell Res. 2021;31(2):107-25.

27. Doll S, Proneth B, Tyurina YY, Panzilius E, Kobayashi S, Ingold I, Irmler M, Beckers J, Aichler M, Walch A, et al. ACSL4 dictates ferroptosis sensitivity by shaping cellular lipid composition. Nat Chem Biol. 2017;13(1):91-8.

28. Chu B, Kon N, Chen D, Li T, Liu T, Jiang L, Song S, Tavana O, Gu W. ALOX12 is required for p53-mediated tumour suppression through a distinct ferroptosis pathway. Nat Cell Biol. 2019;21(5):579-91.

29. Chen X, Comish PB, Tang D, Kang R. Characteristics and Biomarkers of Ferroptosis. Front Cell Dev Biol. 2021;9:637162. 86 e4. 
31. Doll S, Freitas FP, Shah R, Aldrovandi M, da Silva MC, Ingold I, Goya Grocin A, Xavier da Silva TN, Panzilius E, Scheel CH, et al. FSP1 is a glutathione-independent ferroptosis suppressor. Nature. 2019;575(7784):693-8.

32. Brown CW, Amante JJ, Goel HL, Mercurio AM. The alpha6beta4 integrin promotes resistance to ferroptosis. J Cell Biol. 2017;216(12):4287-97.

33. Sun X, Niu X, Chen R, He W, Chen D, Kang R, Tang D. Metallothionein-1G facilitates sorafenib resistance through inhibition of ferroptosis. Hepatology. 2016;64(2):488-500.

34. Zaret KS, Carroll JS. Pioneer transcription factors: establishing competence for gene expression. Genes Dev. 2011;25(21):2227-41.

35. Cirillo LA, Lin FR, Cuesta I, Friedman D, Jarnik M, Zaret KS. Opening of compacted chromatin by early developmental transcription factors HNF3 (FoxA) and GATA-4. Mol Cell. 2002;9(2):279-89.

36. Nakshatri H, Badve S. FOXA1 as a therapeutic target for breast cancer. Expert opinion on therapeutic targets. 2007;11(4):507-14.

37. Gao N, Zhang J, Rao MA, Case TC, Mirosevich J, Wang Y, Jin R, Gupta A, Rennie PS, Matusik RJ. The role of hepatocyte nuclear factor-3 alpha (Forkhead Box A1) and androgen receptor in transcriptional regulation of prostatic genes. Mol Endocrinol. 2003;17(8):1484-507.

38. Moya M, Benet M, Guzman C, Tolosa L, Garcia-Monzon C, Pareja E, Castell JV, Jover R. Foxa1 reduces lipid accumulation in human hepatocytes and is down-regulated in nonalcoholic fatty liver. PLoS One. 2012;7(1):e30014.

39. Slebe F, Rojo F, Vinaixa M, Garcia-Rocha M, Testoni G, Guiu M, Planet E, Samino S, Arenas EJ, Beltran A, et al. FoxA and LIPG endothelial lipase control the uptake of extracellular lipids for breast cancer growth. Nat Commun. 2016;7:11199.

40. BenAyed-Guerfali D, Dabbeche-Bouricha E, Ayadi W, Trifa F, Charfi S, Khabir A, SellamiBoudawara T, Mokdad-Gargouri R. Association of FOXA1 and EMT markers (Twist1 and Ecadherin) in breast cancer. Mol Biol Rep. 2019;46(3):3247-55.

41. Anzai E, Hirata K, Shibazaki M, Yamada C, Morii M, Honda T, Yamaguchi N, Yamaguchi N. FOXA1 Induces E-Cadherin Expression at the Protein Level via Suppression of Slug in Epithelial Breast Cancer Cells. Biol Pharm Bull. 2017;40(9):1483-9.

42. Zhang Y, Zhang D, Li Q, Liang J, Sun L, Yi X, Chen Z, Yan R, Xie G, Li W, et al. Nucleation of DNA repair factors by FOXA1 links DNA demethylation to transcriptional pioneering. Nat Genet. 2016;48(9):1003-13.

43. Lee J, You JH, Kim MS, Roh JL. Epigenetic reprogramming of epithelial-mesenchymal transition promotes ferroptosis of head and neck cancer. Redox Biol. 2020;37:101697.

44. Chen PH, Tseng WH, Chi JT. The Intersection of DNA Damage Response and Ferroptosis-A Rationale for Combination Therapeutics. Biology (Basel). 2020;9(8). 
45. Ferri AL, Lin W, Mavromatakis YE, Wang JC, Sasaki H, Whitsett JA, Ang SL. Foxa1 and Foxa2 regulate multiple phases of midbrain dopaminergic neuron development in a dosage-dependent manner. Development. 2007;134(15):2761-9.

46. Pristera A, Lin W, Kaufmann AK, Brimblecombe KR, Threlfell S, Dodson PD, Magill PJ, Fernandes C, Cragg SJ, Ang SL. Transcription factors FOXA1 and FOXA2 maintain dopaminergic neuronal properties and control feeding behavior in adult mice. Proc Natl Acad Sci U S A. 2015;112(35):E4929-38.

47. Beard JA, Tenga A, Chen T. The interplay of NR4A receptors and the oncogene-tumor suppressor networks in cancer. Cell Signal. 2015;27(2):257-66.

48. Mullican SE, Zhang S, Konopleva M, Ruvolo V, Andreeff M, Milbrandt J, Conneely OM. Abrogation of nuclear receptors $\mathrm{Nr} 4 \mathrm{a} 3$ and $\mathrm{Nr} 4 \mathrm{a} 1$ leads to development of acute myeloid leukemia. Nat Med. 2007;13(6):730-5.

49. Ramirez-Herrick AM, Mullican SE, Sheehan AM, Conneely OM. Reduced NR4A gene dosage leads to mixed myelodysplastic/myeloproliferative neoplasms in mice. Blood. 2011;117(9):2681-90.

50. Sekiya T, Kashiwagi I, Yoshida R, Fukaya T, Morita R, Kimura A, Ichinose H, Metzger D, Chambon P, Yoshimura A. Nr4a receptors are essential for thymic regulatory T cell development and immune homeostasis. Nat Immunol. 2013;14(3):230-7.

51. Odagiu L, Boulet S, Maurice De Sousa D, Daudelin JF, Nicolas S, Labrecque N. Early programming of CD8(+) T cell response by the orphan nuclear receptor NR4A3. Proc Natl Acad Sci U S A. 2020;117(39):24392-402.

52. Wang H, Meyer CA, Fei T, Wang G, Zhang F, Liu XS. A systematic approach identifies FOXA1 as a key factor in the loss of epithelial traits during the epithelial-to-mesenchymal transition in lung cancer. BMC Genomics. 2013;14:680.

53. Skene PJ, Henikoff S. An efficient targeted nuclease strategy for high-resolution mapping of DNA binding sites. Elife. 2017;6.

54. Meers MP, Tenenbaum D, Henikoff S. Peak calling by Sparse Enrichment Analysis for CUT\&RUN chromatin profiling. Epigenetics Chromatin. 2019;12(1):42.

55. De Cecco M, Criscione SW, Peckham EJ, Hillenmeyer S, Hamm EA, Manivannan J, Peterson AL, Kreiling JA, Neretti N, Sedivy JM. Genomes of replicatively senescent cells undergo global epigenetic changes leading to gene silencing and activation of transposable elements. Aging Cell. 2013;12(2):247-56.

56. Tasselli L, Xi Y, Zheng W, Tennen RI, Odrowaz Z, Simeoni F, Li W, Chua KF. SIRT6 deacetylates $\mathrm{H} 3 \mathrm{~K} 18 \mathrm{ac}$ at pericentric chromatin to prevent mitotic errors and cellular senescence. Nat Struct Mol Biol. 2016;23(5):434-40.

57. Zhou Y, Zhou B, Pache L, Chang M, Khodabakhshi AH, Tanaseichuk O, Benner C, Chanda SK. Metascape provides a biologist-oriented resource for the analysis of systems-level datasets. Nat Commun. 2019;10(1):1523. 
58. Pachkov M, Balwierz PJ, Arnold P, Ozonov E, van Nimwegen E. SwissRegulon, a database of genome-wide annotations of regulatory sites: recent updates. Nucleic Acids Res. 2013;41(Database issue):D214-20.

59. Human transcription factor target genes. https://github.com/slowkow/tftargets. Date accessed: 18th May 2021

60. Alim I, Caulfield JT, Chen Y, Swarup V, Geschwind DH, Ivanova E, Seravalli J, Ai Y, Sansing LH, Ste Marie EJ, et al. Selenium Drives a Transcriptional Adaptive Program to Block Ferroptosis and Treat Stroke. Cell. 2019;177(5):1262-79 e25.

61. Ayala A, Munoz MF, Arguelles S. Lipid peroxidation: production, metabolism, and signaling mechanisms of malondialdehyde and 4-hydroxy-2-nonenal. Oxidative medicine and cellular longevity. 2014;2014:360438.

62. Chen PH, Wu J, Ding CC, Lin CC, Pan S, Bossa N, Xu Y, Yang WH, Mathey-Prevot B, Chi JT. Kinome screen of ferroptosis reveals a novel role of ATM in regulating iron metabolism. Cell Death Differ. 2020;27(3):1008-22.

63. Tan NY, Khachigian LM. Sp1 phosphorylation and its regulation of gene transcription. Mol Cell Biol. 2009;29(10):2483-8.

64. Ye Z, Zhuo Q, Hu Q, Xu X, Mengqi L, Zhang Z, Xu W, Liu W, Fan G, Qin Y, et al. FBW7NRA41-SCD1 axis synchronously regulates apoptosis and ferroptosis in pancreatic cancer cells. Redox Biol. 2021;38:101807.

65. Safe S, Shrestha R, Mohankumar K. Orphan nuclear receptor 4A1 (NR4A1) and novel ligands. Essays Biochem. 2021.

66. Wu H, Wang Y, Tong L, Yan H, Sun Z. Global Research Trends of Ferroptosis: A Rapidly Evolving Field With Enormous Potential. Front Cell Dev Biol. 2021;9:646311.

67. Chu S. Transcriptional regulation by post-transcriptional modification--role of phosphorylation in Sp1 transcriptional activity. Gene. 2012;508(1):1-8.

68. Hattori K, Ishikawa H, Sakauchi C, Takayanagi S, Naguro I, Ichijo H. Cold stress-induced ferroptosis involves the ASK1-p38 pathway. EMBO Rep. 2017;18(11):2067-78.

69. Li L, Hao Y, Zhao Y, Wang H, Zhao X, Jiang Y, Gao F. Ferroptosis is associated with oxygenglucose deprivation/reoxygenation-induced Sertoli cell death. Int J Mol Med. 2018;41(5):305162.

70. Yu Y, Xie Y, Cao L, Yang L, Yang M, Lotze MT, Zeh HJ, Kang R, Tang D. The ferroptosis inducer erastin enhances sensitivity of acute myeloid leukemia cells to chemotherapeutic agents. Mol Cell Oncol. 2015;2(4):e1054549.

71. Kwon OS, Kwon EJ, Kong HJ, Choi JY, Kim YJ, Lee EW, Kim W, Lee H, Cha HJ. Systematic identification of a nuclear receptor-enriched predictive signature for erastin-induced ferroptosis. Redox Biol. 2020;37:101719. 

$\mathrm{K}$, Wierman ME, Angeli JPF, et al. Active steroid hormone synthesis renders adrenocortical cells highly susceptible to type II ferroptosis induction. Cell Death Dis. 2020;11(3):192.

73. Belavgeni A, Bornstein SR, Linkermann A. Stress will kill you anyway! Cell Death Dis. 2020;11(4):218.

74. Augello MA, Hickey TE, Knudsen KE. FOXA1: master of steroid receptor function in cancer. EMBO J. 2011;30(19):3885-94.

75. Hertz R, Magenheim J, Berman I, Bar-Tana J. Fatty acyl-CoA thioesters are ligands of hepatic nuclear factor-4alpha. Nature. 1998;392(6675):512-6.

76. Kang R, Kroemer G, Tang D. The tumor suppressor protein p53 and the ferroptosis network. Free Radic Biol Med. 2019;133:162-8.

77. Fedorova O, Petukhov A, Daks A, Shuvalov O, Leonova T, Vasileva E, Aksenov N, Melino G, Barlev NA. Orphan receptor NR4A3 is a novel target of p53 that contributes to apoptosis. Oncogene. 2019;38(12):2108-22.

78. Viswanathan VS, Ryan MJ, Dhruv HD, Gill S, Eichhoff OM, Seashore-Ludlow B, Kaffenberger SD, Eaton JK, Shimada K, Aguirre AJ, et al. Dependency of a therapy-resistant state of cancer cells on a lipid peroxidase pathway. Nature. 2017;547(7664):453-7.

79. Hangauer MJ, Viswanathan VS, Ryan MJ, Bole D, Eaton JK, Matov A, Galeas J, Dhruv HD, Berens ME, Schreiber SL, et al. Drug-tolerant persister cancer cells are vulnerable to GPX4 inhibition. Nature. 2017;551(7679):247-50.

80. Metzakopian E, Bouhali K, Alvarez-Saavedra M, Whitsett JA, Picketts DJ, Ang SL. Genomewide characterisation of Foxa1 binding sites reveals several mechanisms for regulating neuronal differentiation in midbrain dopamine cells. Development. 2015;142(7):1315-24.

81. Smurova K, De Wulf P. Centromere and Pericentromere Transcription: Roles and Regulation ... in Sickness and in Health. Front Genet. 2018;9:674.

82. Eymery A, Callanan M, Vourc'h C. The secret message of heterochromatin: new insights into the mechanisms and function of centromeric and pericentric repeat sequence transcription. Int J Dev Biol. 2009;53(2-3):259-68.

83. Hall LE, Mitchell SE, O'Neill RJ. Pericentric and centromeric transcription: a perfect balance required. Chromosome Res. 2012;20(5):535-46.

84. Jolly C, Metz A, Govin J, Vigneron M, Turner BM, Khochbin S, Vourc'h C. Stress-induced transcription of satellite III repeats. J Cell Biol. 2004;164(1):25-33.

85. Valgardsdottir R, Chiodi I, Giordano M, Rossi A, Bazzini S, Ghigna C, Riva S, Biamonti G. Transcription of Satellite III non-coding RNAs is a general stress response in human cells. Nucleic Acids Res. 2008;36(2):423-34.

86. Rizzi N, Denegri M, Chiodi I, Corioni M, Valgardsdottir R, Cobianchi F, Riva S, Biamonti G. Transcriptional activation of a constitutive heterochromatic domain of the human genome in response to heat shock. Mol Biol Cell. 2004;15(2):543-51. 
87. Li Q, Zhang Y, Fu J, Han L, Xue L, Lv C, Wang P, Li G, Tong T. FOXA1 mediates p16(INK4a) activation during cellular senescence. EMBO J. 2013;32(6):858-73.

88. Hirata K, Takakura Y, Shibazaki M, Morii M, Honda T, Oshima M, Aoyama K, Iwama A, Nakayama Y, Takano H, et al. Forkhead box protein A1 confers resistance to transforming growth factor-beta-induced apoptosis in breast cancer cells through inhibition of Smad3 nuclear translocation. J Cell Biochem. 2018.

89. Palagani A, Op de Beeck K, Naulaerts S, Diddens J, Sekhar Chirumamilla C, Van Camp G, Laukens K, Heyninck K, Gerlo S, Mestdagh P, et al. Ectopic microRNA-150-5p transcription sensitizes glucocorticoid therapy response in MM1S multiple myeloma cells but fails to overcome hormone therapy resistance in MM1R cells. PLoS One. 2014;9(12):e113842.

90. Logie E, Chirumamilla CS, Perez-Novo C, Shaw P, Declerck K, Palagani A, Rangarajan S, Cuypers B, De Neuter N, Mobashar Hussain Urf Turabe F, et al. Covalent Cysteine Targeting of Bruton's Tyrosine Kinase (BTK) Family by Withaferin-A Reduces Survival of GlucocorticoidResistant Multiple Myeloma MM1 Cells. Cancers (Basel). 2021;13(7).

91. FastQC. 2015

92. Dobin A, Davis CA, Schlesinger F, Drenkow J, Zaleski C, Jha S, Batut P, Chaisson M, Gingeras TR. STAR: ultrafast universal RNA-seq aligner. Bioinformatics. 2013;29(1):15-21.

93. Love MI, Huber W, Anders S. Moderated estimation of fold change and dispersion for RNA-seq data with DESeq2. Genome Biol. 2014;15(12):550.

94. Zhang X, Du L, Qiao Y, Zhang X, Zheng W, Wu Q, Chen Y, Zhu G, Liu Y, Bian Z, et al. Ferroptosis is governed by differential regulation of transcription in liver cancer. Redox Biol. 2019;24:101211.

95. Rueden CT, Schindelin J, Hiner MC, DeZonia BE, Walter AE, Arena ET, Eliceiri KW. ImageJ2: ImageJ for the next generation of scientific image data. BMC Bioinformatics. 2017;18(1):529.

96. Pachkov M, Erb I, Molina N, van Nimwegen E. SwissRegulon: a database of genome-wide annotations of regulatory sites. Nucleic Acids Res. 2007;35(Database issue):D127-31.

97. Chen EY, Xu H, Gordonov S, Lim MP, Perkins MH, Ma'ayan A. Expression2Kinases: mRNA profiling linked to multiple upstream regulatory layers. Bioinformatics. 2012;28(1):105-11.

98. Skene PJ, Henikoff JG, Henikoff S. Targeted in situ genome-wide profiling with high efficiency for low cell numbers. Nat Protoc. 2018;13(5):1006-19.

99. Li H. Aligning sequence reads, clone sequences and assembly contigs with BWA-MEM. 2013.

100. Quinlan AR and Hall IM. BEDTools: a flexible suite of utilities for comparing genomic features. Bioinformatics. 2010;16:841-842. Software for computing and annotating genomic ranges. PLoS Computational Biology. 2013;9. associated accessibility from single-cell epigenomic data. Nature Methods. 2017;14:975-978. 
bioRxiv preprint doi: https://doi.org/10.1101/2021.10.13.461056; this version posted October 14,2021 . The copyright holder for this

preprint (which was not certified by peer review) is the author/funder, who has granted bioRxiv a license to display the preprint in perpetuity. It is made available under aCC-BY-ND 4.0 International license.

809 103. Zhang H, Meltzer P, Davis S. RCircos: an R package for Circos 2D track plots. BMC

$810 \quad$ Bioinformatics. 2013;14:244. 